INPLASY

PROTOCOL

To cite: Xu et al. Efficacy and safety of High power short duration Catherter ablation for Atrial fibrillation: an updated meta-analysis. Inplasy protocol 2020120069. doi:

10.37766/inplasy2020.12.0069

Received: 12 December 2020

Published: 12 December 2020

Corresponding author:

Min Xu

286412861@qq.com

Author Affiliation:

The Second Affiliated

Hospital of North Sichuan

Medical College (Sichuan

Mianyang 404 Hospital)

Support: Health Commission of Sichuan P.

Review Stage at time of this submission: The review has not yet started.

Conflicts of interest: None.

\section{Efficacy and safety of High power short duration Catherter ablation for Atrial fibrillation: an updated meta- analysis}

\author{
$\mathrm{Xu}, \mathrm{M} 1$; Yang, Y2; Zhang, DY3.
}

Review question / Objective: The population, intervention, comparison, and outcome (PICO) approach was adapted. The patients with AF (paroxysmal AF and persistent AF) were included in population interest. The intervention was Pulmonary vein isolation (tadditional lesions) via RFCA (radiofrequency catheter ablation) in the treatment of atrial fibrillation. The comparison of interest was the ablation energy: High power short duration vs. Low power long duration. The primary outcome assessment was atrial arrhythmia recurrence post-blanking period, first-pass PV isolation and acute PV reconnection. The secondary outcomes included procedure time, fluoroscopy time, RF time, ablation time and procedure complication.

Condition being studied: In recent years, catheter ablation is the most effective therapy for symptomatic af, and recommend by the relevant guidelines. But recurrence rates are still high after catheter ablation. High power short duration strategy is a hot research spot, is expected to further improve the long-term clinincal outcomes of atrial arrathymiafree survival after catheter ablation. In recent two years, more than 10 clinical studies assessed that HPSD clinical effect. But the verdict is mixed.

INPLASY registration number: This protocol was registered with the International Platform of Registered Systematic Review and Meta-Analysis Protocols (INPLASY) on 12 December 2020 and was last updated on 12 December 2020 (registration number INPLASY2020120069).

\section{INTRODUCTION}

Review question / Objective: The population, intervention, comparison, and outcome (PICO) approach was adapted. The patients with AF (paroxysmal AF and persistent AF) were included in population interest. The intervention was Pulmonary vein isolation ( \pm additional lesions) via RFCA (radiofrequency catheter ablation) in the treatment of atrial fibrillation. The comparison of interest was the ablation 
energy: High power short duration vs. Low power long duration. The primary outcome assessment was atrial arrhythmia recurrence post-blanking period, first-pass PV isolation and acute PV reconnection. The secondary outcomes included procedure time, fluoroscopy time, RF time, ablation time and procedure complication.

Rationale: Updated clinical studies in pubmedM, Cochrane, Medline and other databases were searched, and the clinical outcomes of HPSD (High power short duration) in radiofrequency catheter ablation for atrial fibrillation was studied via meta analysis. Analyze the main reasons for the difference in outcome.

Condition being studied: In recent years, catheter ablation is the most effective therapy for symptomatic af, and recommend by the relevant guidelines. But recurrence rates are still high after catheter ablation. High power short duration strategy is a hot research spot, is expected to further improve the long-term clinincal outcomes of atrial arrathymia-free survival after catheter ablation. In recent two years, more than 10 clinical studies assessed that HPSD clinical effect. But the verdict is mixed.

\section{METHODS}

Search strategy: Boolean logic operators (AND, OR, NOT) were used to search for keywords and free words. The keywords included "High power short duration", "Ablation index", "Atrial Fibrillation", "Catheter Ablation", "Radiofrequency ablation", etc.

Participant or population: Age 18-80. At least 1 ECG and/or Holer diagnosis of paroxysmal/persistent atrial fibrillation in the last 6 months. Non-valvular patients. Regardless of prior radiofrequency ablation history or not.

Intervention: Radiofrequency catheter radiofrequency.

Comparator: High power ( $>40 W$ ) short duration.
Study designs to be included: Randomised controlled trial (RCT), self-controlled studies, one arm studies, and cohort studies (CS).

Eligibility criteria: (1)Objective to study the clinical outcomes of HPSD on AF (2)Controlled study (3)Balance of baseline data (4)The studies include the main outcome or secondary outcome mentioned above (5)Public publication.

Information sources: The databases included PubMed, Cochrane, EMbase, Medline and the chinese medical database(e.g. Wanfang database, China National Knowledge Infrastructure (CNKI), CQVIP, CBM library). The language and the time of studies included was not limited.

Main outcome(s): Atrial arrhythmia recurrence post-blanking period, first-pass $P V$ isolation and acute PV reconnection.

Additional outcome(s): Procedure time, fluoroscopy time, RF time, ablation time and procedure complication.

Quality assessment / Risk of bias analysis: Delphi List would be applied in randomised controlled trial. The Newcastle-Ottawa Scale (NOS) would be adapted to evaluating case-control studies and cohort studies.

Strategy of data synthesis: RevMan version 5.3 software was applied in this study. Actual incidence frequency would be extracted for statistics in ATAs recurrence, procedure complication, Acute PVR and PFI. The weighted mean difference and $95 \%$ confidence interval would be applied for statistical analysis in procedure time, fluoroscopy time, RF time, time and number for PVI. When $12>75 \%$, the fixed effect model would be applied to calculate the pooled RR.When $12>50 \%$ and $<75 \%$, the random effect model would be applied to calculate RR/OR. The potential publication bias would be detected by funnel plot.

Subgroup analysis: If data volume supports, paroxysmal and persistent AF 
data above will be presented in the subgroup analysis.

Sensibility analysis: The outcomes would be evaluated according to GRADE (Grading of Recommendations Assessment, Development and Evaluation). The evidence levels would be classified into four levels: high, moderate, low, and very low.

Language: The language would not be limited.

Country(ies) involved: China.

Keywords: High power short duration; atrial fibrillation; radiofrequency ablation; pulmonary vein isolation; ablation index; meta-analysis.

Contributions of each author:

Author 1 - Min Xu - The author drafted the manuscript.

Author 2 - Yang Yan - The author was responsible data extraction and quality assessment.

Author 3 - Zhang Dayong - The author was responsible data extraction and quality assessment. 